\title{
CAIR-1/BAG-3 modulates cell adhesion and migration by downregulating activity of focal adhesion proteins
}

\author{
Jareer N. Kassis ${ }^{a, *}$, Elizabeth A. Guancial ${ }^{b}$, Howard Doong ${ }^{a, 1}$, \\ Victoria Virador ${ }^{a}$, Elise C. Kohn ${ }^{a}$ \\ ${ }^{a}$ Molecular Signaling Section, Laboratory of Pathology, Center for Cancer Research, National Cancer Institute, 10 Center Drive, MSC 1500 , \\ Bethesda, MD 20892-1500, USA \\ ${ }^{\mathrm{b}}$ Howard Hughes Medical Institute/NIH Research Scholar, Bethesda, MD 20892, USA
}

\section{A R T I C L E I N F O R M A T I O N}

Article Chronology:

Received 18 August 2005

Revised version received

19 May 2006

Accepted 31 May 2006

Available online 21 June 2006

\section{Keywords:}

FAK

Paxillin

Collagen type IV

Signalosome

\begin{abstract}
A B S T R A C T
CAIR-1/BAG-3 is a stress and survival protein that has been shown to bind SH3 domaincontaining proteins through its proline-rich (PXXP) domain. Because stress and survival pathways are active during invasion and metastasis, we hypothesized that CAIR-1 is a regulator of signaling pathways that modulate cell adhesion and migration. MDA-435 human breast carcinoma cells were stably transfected with full-length CAIR-1 (FL) or a proline-rich domain deleted mutant (dPXXP). FL cells migrated poorly through collagen IV-coated filters to serum (14\% of control, $p=0.0004)$, whereas migration of dPXXP cells was more robust (228\%, $p=0.00001)$. Adhesion to collagen IV-coated surfaces was reduced in FL cells and augmented in dPXXP cells (FL 64\%, $p=0.03$; dPXXP 138\%, $p=0.01$ ). Rhodamine-phalloidin staining highlighted more stress fibers and thicker filopodial protrusions in dPXXP cells. Fewer focal adhesions were also seen in FL cells. A reduction in tyrosine phosphorylation of focal adhesion kinase (FAK) and paxillin occurred in FL cells under these conditions. In contrast, increased FAK and paxillin phosphorylation was documented in dPXXP cells. Differential FAK phosphorylation occurred at the major autophosphorylation site $\mathrm{Y}^{397}$ and Src phosphorylation site $\mathrm{Y}^{861}$. Concordant with these findings, there was decreased interaction between FAK and its downstream partners $\mathrm{p}^{130}$ Cas and Crk observed in FL cells but not in dPXXP cells. These results collectively indicate that CAIR-1 may negatively regulate adhesion, focal adhesion assembly, signaling, and migration via its PXXP domain.
\end{abstract}

Published by Elsevier Inc.

\section{Introduction}

Cell migration is a dynamic process governed by intra- and extracellular stimuli that promote the formation of focal adhesions between the cell membrane and the extracellular matrix (ECM) [1]. Migration involves dynamic remodeling of focal adhesions and interaction with the extracellular microenvironment. Dysregulated migration is a hallmark of tumor cell invasion and progression towards metastasis, resulting in poor patient prognosis [2,3]. Overexpression of key proteins in migration, such as receptor tyrosine kinases, integrins, and their downstream effector molecules, can

\footnotetext{
* Corresponding author. Fax: +1 3014805142 .

E-mail address: kassisj@mail.nih.gov (J.N. Kassis).

${ }^{1}$ Present address: University of Maryland, UMBI, Medical Biotechnology Center, 725 W. Lombard St. N355, Baltimore, MD 21201 , USA.
} 
promote dysregulated migration and support tumor invasion [4]. Therefore, understanding the regulatory elements that control signaling of attachment and migration is critical to advancing cancer intervention.

CAIR-1/BAG-3 [5,6] is a stress- and survival-related protein that was shown to be upregulated upon prolonged cell exposure to CAI, an inhibitor of non-voltage regulated calcium influx and calcium-regulated adhesion and motility [7-9]. CAIR-1 has three distinct protein interaction motifs: a WW domain, a proline-rich (PXXP) domain, and a BAG domain [9]. The BAG domain binds to HSP 70, whereas the proline-rich (PXXP) region binds SH3 domains such as in PLC $\gamma$. SH3 interactions have been shown to be important in invasion and tumor progression [10, 11]. This suggested that CAIR-1 might be a chaperone or regulatory protein for those $\mathrm{SH} 3$ proteins involved in cell migration or adhesion.

Focal adhesion kinase (FAK) is a major component of focal adhesions formed by the leading edge of a migrating or attaching cell $[12,13]$. Focal adhesions are points of high-strength attachment of the cell to the ECM, and each constitutes a highly active signalosome comprised of a cluster of adhesion signaling proteins and receptors [1,14]. FAK is activated by integrin engagement and/or by activation of receptor tyrosine kinases, leading to downstream stimulation of non-receptor kinases. FAK interacts with other migration-associated proteins such as Src and paxillin. Activated FAK binds Src to produce downstream signaling events involving paxillin as well as SH3 domaincontaining proteins such as $\mathrm{p}^{130}$ Cas $[15,16]$. Elucidation of the regulatory mechanisms that govern interactions of these proteins with each other as well as their recruitment to focal adhesions will enhance our understanding of signaling events that promote dysregulated adhesion and migration in cancer cells.

We hypothesized that CAIR-1 modulates the migratory cell phenotype through interaction with SH3 domain-containing proteins involved in the signaling of adhesion and migration. We stably transfected MDA-435 human breast carcinoma cells with either full-length CAIR-1 [6] or a truncated construct with a deleted PXXP domain. We report that overexpression of fulllength CAIR-1 significantly reduces adhesion and migration and results in decreased focal adhesion signaling. These results point to a novel role for CAIR-1 in signaling of cell adhesion and migration.

\section{Materials and methods}

\section{Cell culture}

MDA-MB-435 human breast cancer cells, an invasive estrogen receptor-negative line [17], were obtained from ATCC (Manassas, VA) and were maintained in DMEM with 10\% fetal calf serum (FCS). Full-length (FL) CAIR-1 constructs were generated with a (His) 6 tag at the $\mathrm{N}$ terminus by PCR cloning as described previously [6]. CAIR-1 with a prolinerich domain deletion mutant (a.a. 319-423) was constructed using the $5^{\prime}$ (His) $)_{6}$-containing primer and $3^{\prime}$ terminal oligonucleotides previously reported. An internal HindIII ligation site that did not alter the protein sequence was engineered at the deletion, using primers shown in Fig. 1A. Sequence validated DNA was transfected into MDA-MB-435 cells using Lipofectamine reagent (Invitrogen, Carsbad, CA) according to manufacturer's instructions. Cells were selected, expanded, and maintained in $800 \mu \mathrm{g} / \mathrm{ml} \mathrm{G418}$ except for the passage immediately prior to experiments. Multiple clones of each subline were developed, and we verified that at least two phenotypes described in this study (adhesion and FAK phosphorylation) were consistent across different clones of the same variants. Expression of CAIR-1 on the wild-type background was confirmed regularly by Western blotting (Fig. 1B). In all experiments, cells were grown to semi-confluence, as previous observations showed a downregulation of wild-type CAIR-1 protein expression associated with full confluency.

\section{Immunoprecipitation and immunoblotting}

Cells were lysed in modified RIPA buffer (50 mM Tris-HCl pH 7.5, $1 \%$ NP-40, $0.1 \%$ sodium deoxycholate, $150 \mathrm{mMNaCl}, 4 \mathrm{mMEDTA}$, $10 \mathrm{mM}$ sodium pyrophosphate, $2 \mathrm{mM}$ sodium orthovanadate,

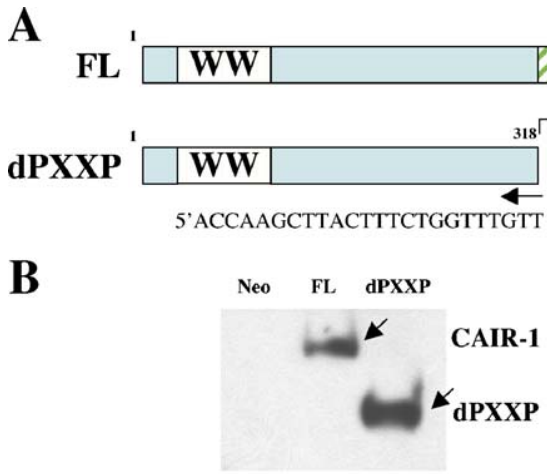

IB: Anti-(His) $)_{6}$
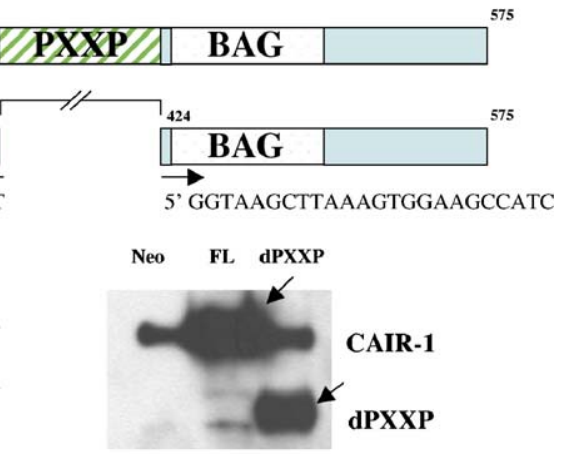

IB: Anti-CAIR-1

Fig. 1 - CAIR-1 expression. (A) Diagram of CAIR-1/BAG-3 illustrating domains of interest. Primers used to construct dPXXP CAIR-1 are indicated. (B) MDA-435 cell sublines were stably transfected with FL or dPXXP CAIR-1 mutants containing a (His) 6 tag. Cells lysates were subjected to immunoblotting and probed with either anti-His antibody, left panel, or anti-CAIR-1 antibody, right panel (also illustrating endogenous expression). Arrows point to expressed constructs. 
$100 \mathrm{mM}$ phenylmethyl-sulphonylfluoride (PMSF), $10 \mu \mathrm{g} / \mathrm{ml}$ each of aprotinin and leupeptin). Protein content was determined using a BCA protein assay kit (Pierce, Rockford, IL). Lysate $(500 \mu \mathrm{g}$ protein) was incubated with primary antibody at $4^{\circ} \mathrm{C}$ for $4 \mathrm{~h}$ for immunoprecipitation; protein $\mathrm{A} / \mathrm{G}$ beads (Pierce) were added during the last $2 \mathrm{~h}$ of incubation. Bound proteins were separated by SDS-PAGE and transferred to a PVDF membrane that was blocked in $5 \%$ nonfat milk plus $1 \%$ ovalbumin, and then probed by immunoblotting. Antibodies for Western blotting included His (Applied Biological Materials, Richmond, BC), FAK, Src, and paxillin (Upstate Biotechnology; Charlottesville, VA), $\mathrm{p}^{130} \mathrm{Cas}$ and Crk (BD Transduction Labs, San Jose, CA), phospho-FAK pY $^{397}$, phospho-FAK pY ${ }^{861}$, and phospho-Src pY ${ }^{418}$ (Biosource; Camarillo, CA), $\beta$-tubulin (Abcam; Cambridge, MA), and polyclonal CAIR-1 antibodies [6].

\section{Boyden chamber migration assays}

Boyden chambers and PVP-free polycarbonate membranes with $8-\mu \mathrm{m}$ pores were obtained from NeuroProbe, Inc. (Gaithersburg, MD). The membranes were coated with a solution of $100 \mu \mathrm{g} / \mathrm{ml}$ human collagen IV (Becton Dickinson, Franklin Lakes, NJ) in $0.1 \%$ acetic acid at $4^{\circ} \mathrm{C}$. Cells were harvested and allowed $15 \mathrm{~min}$ to recover in suspension in serum-containing medium at room temperature, then pelleted and resuspended in DMEM containing 0.1\% BSA (Fraction $\mathrm{V}, 2 \times 10^{6} \mathrm{cells} / \mathrm{ml}$ ). DMEM with $10 \%$ FCS was used in the bottom chamber as a chemoattractant, whereas DMEM with $0.1 \%$ BSA (Fraction V) was used as a negative control. For every experiment, each treatment and subline was tested in triplicate. The chambers were incubated overnight, after which cells were fixed in methanol and stained in Dif-Quik solution (VWR, West Chester, PA). Non-migrated cells were removed and five random fields from each pellet were counted at 40x magnification. Each experiment was done at least twice for each batch of collagen IV-coated filters.

\section{Adhesion assays}

Cytomatrix extracellular matrix-coated 96-well chambers were purchased from Chemicon (Temecula, CA) and assays were performed according to manufacturer's instructions. Cells in $10 \%$ FCS-containing media $\left(5 \times 10^{4}\right.$ per well $)$ were incubated in duplicate wells for $3 \mathrm{~h}$ at $37^{\circ} \mathrm{C}$; $3 \mathrm{~h}$ was found optimal for these cells to produce focal adhesions and only in the presence of serum. Adherent cells were fixed in a $0.1 \%$ crystal violet $/ 10 \%$ ethanol solution for $5 \mathrm{~min}$, washed, and bound dye was eluted with a 1:1 solution of $50 \%$ ethanol: $0.1 \mathrm{M} \mathrm{NaH}_{2} \mathrm{PO}_{4}(\mathrm{pH} 4.45)$. The absorbance of the eluted solution was read at $540 \mathrm{~nm}$. Each of these assays was performed at least four times per substrate.

\section{Calpain activity assays}

Calpain activity was assessed using the Boc assay method with a fluorescent calpain substrate to reflect calpain activity [18]. Cells were plated on collagen IV $(25 \mathrm{nM})$ pre-coated glass coverslips and allowed to attach for $3 \mathrm{~h}$. Adherent cells were treated with $1 \mu \mathrm{M}$ t-butoxycarbonyl-Leu-Met-7-amino-4chloromethyl-coumarin (BOC-Leu-Met-CMAC; Molecular Probes, Eugene, OR) during the final $30 \mathrm{~min}$ of attachment. Coverslips were wet-mounted onto glass slides and observed at $353 \mathrm{~nm}$ with a fluorescent microscope. These assays were performed three times.

\section{Immunofluorescence}

Cells were allowed to attach for $3 \mathrm{~h}$ to glass coverslips precoated with $25 \mathrm{nM}$ collagen IV. They were then fixed in $3.7 \%$ formaldehyde in PBS, permeabilized with $0.5 \%$ Triton X-100, and blocked with 1\% BSA (Fraction V) in PBS for $1 \mathrm{~h}$. Coverslips were incubated with the indicated antibodies (Upstate Biotechnology, Charlottesville, VA) at a 1:500 dilution, or anti CAIR-1 at a 1:200 dilution in PBS overnight at $4^{\circ} \mathrm{C}$, or with

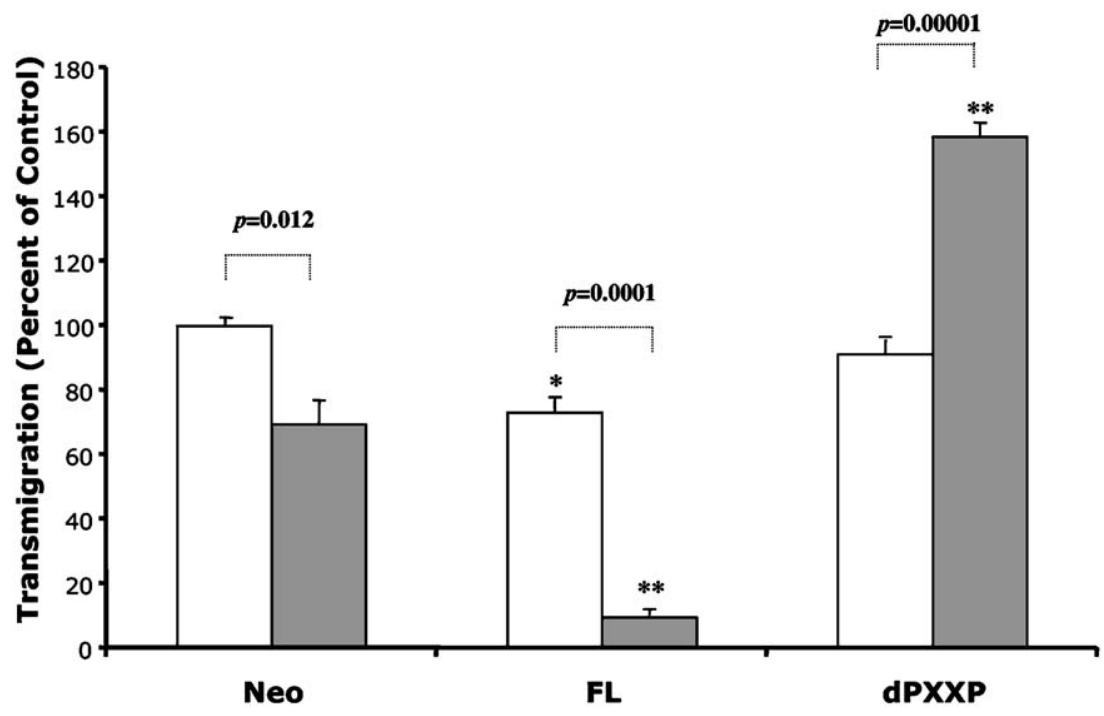

Fig. 2 - Overexpression of CAIR-1 inhibits migration to serum. Cells were suspended in $0.1 \%$ BSA media and allowed to migrate to $10 \%$ FCS media through a collagen IV-coated filter. White bars indicate migration to $0.1 \%$ BSA control, shaded bars denote migration to $10 \%$ FCS, \pm SEM. ${ }^{*} p<0.01$ compared to Neo in serum-free medium; ${ }^{* *} p<0.001$ compared to Neo in serum containing medium. 
Table 1 - Adhesion of MDA-435 sublines to different substrata \pm SEM, $n=4$

\begin{tabular}{lcc} 
Substrate & $\begin{array}{c}\text { FL (percentage of } \\
\text { Neo, } p \text { value) }\end{array}$ & $\begin{array}{c}\text { dPXXP (percentage of } \\
\text { Neo, } p \text { value) }\end{array}$ \\
\hline Collagen IV & $64 \pm 12,0.027$ & $138 \pm 11,0.010$ \\
Collagen I & $68 \pm 19, \mathrm{NS}$ & $149 \pm 25, \mathrm{NS}$ \\
Fibronectin & $72 \pm 16, \mathrm{NS}$ & $127 \pm 15, \mathrm{NS}$ \\
Vitronectin & $45 \pm 9,0.009$ & $114 \pm 14, \mathrm{NS}$ \\
Laminin & $51 \pm 12,0.024$ & $122 \pm 10, \mathrm{NS}$ \\
\hline
\end{tabular}

NS-not significant.

rhodamine-phalloidin (Molecular Probes, Eugene, OR) at a 1:40 dilution in PBS for $20 \mathrm{~min}$. Antibody-treated cells were washed in PBS and incubated with Alexa-Fluor 594 goat antimouse or Alexa-Fluor 488 anti-rabbit secondary antibodies (Molecular Probes, Eugene, OR) at a 1:500 dilution in PBS for 1h. Coverslips were washed and mounted using Vectashield with DAPI (Vector Labs, Burlingame, CA). Cells probed with rhodamine-phalloidin were washed and immediately mounted and observed with confocal fluorescent microscopy. Quantitation was done on experiments for which at least five different fields containing at least 3 cells each were photographed. All focal adhesions on at least three randomly selected cells (up to 12 cells depending on number visible in each field) were counted and tabulated $(n=2)$.

\section{Gene silencing}

BAG-3 and control (non-silencing) siRNA labeled at the $3^{\prime}$ end with Alexa Fluor 488 were purchased from QIAGEN, Inc. (Valencia, CA). Two hundred nanomolar of BAG-3 or nonsilencing siRNA was suspended in serum-free DMEM with $8 \mu \mathrm{l} / \mathrm{ml}$ siLentFect ${ }^{\mathrm{TM}}$ lipid reagent (Bio-Rad, Hercules, CA) for

A

Neo
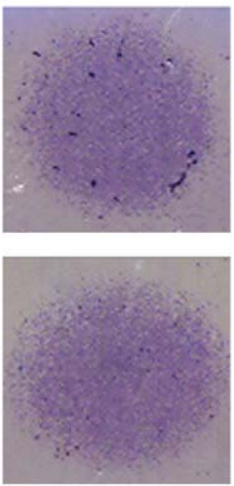

FL
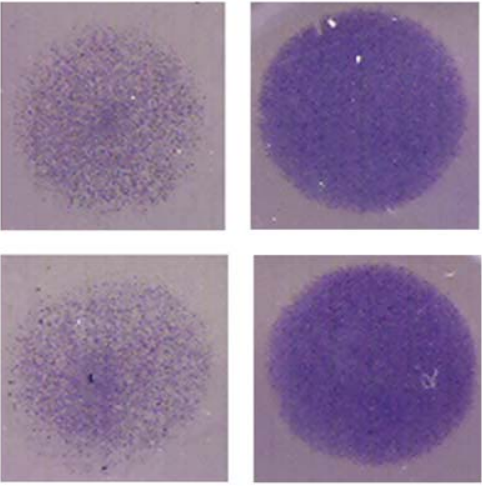

dPXXP

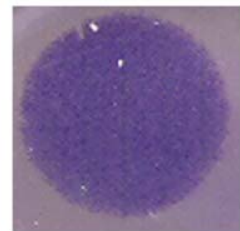

B
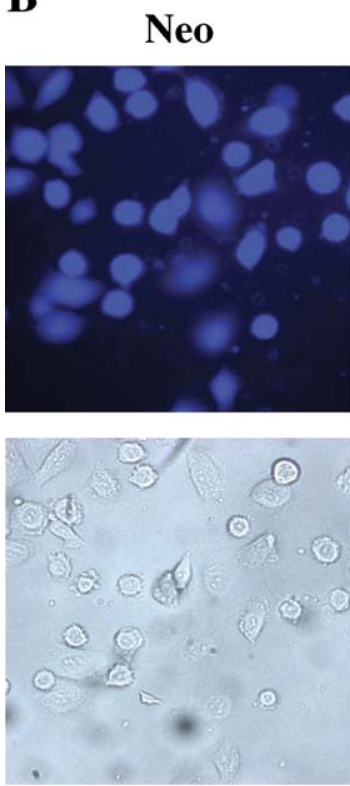

FL
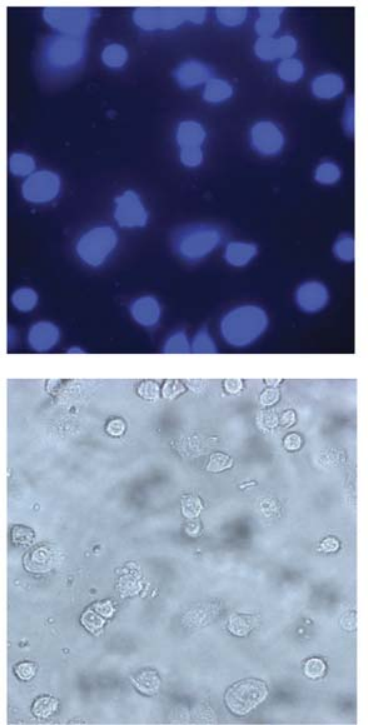
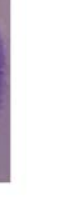
$20 \mathrm{~min}$. The mixture was then aliquoted into 6-well plates containing pro-plated cells to a final concentration of $40 \mathrm{nM}$ of siRNA per well. Forty-eight hours later, cells were either lysed for Western blotting or transferred onto collagen IVcoated coverslips where they were incubated for $3 \mathrm{~h}$ prior to fixing and staining as described in the Immunofluorescence section.

\section{Statistical analysis}

Student's $t$ test analysis was done using Microsoft ${ }^{\circledR}$ Excel (Redmond, WA). All reported $P$ values are two-tailed.

\section{Results}

Overexpression of CAIR-1 decreases migration and attachment

We hypothesized that CAIR-1 plays a role in regulating signaling of migration and adhesion pathways. MDA-435 cells overexpressing full-length CAIR-1 (FL), CAIR-1 with a deleted proline-rich domain (dPXXP), and empty vector cells (Neo; Fig. 1) were exposed to $10 \%$ serum in medium as the chemoattractant in modified Boyden chamber assays using
A

FAK
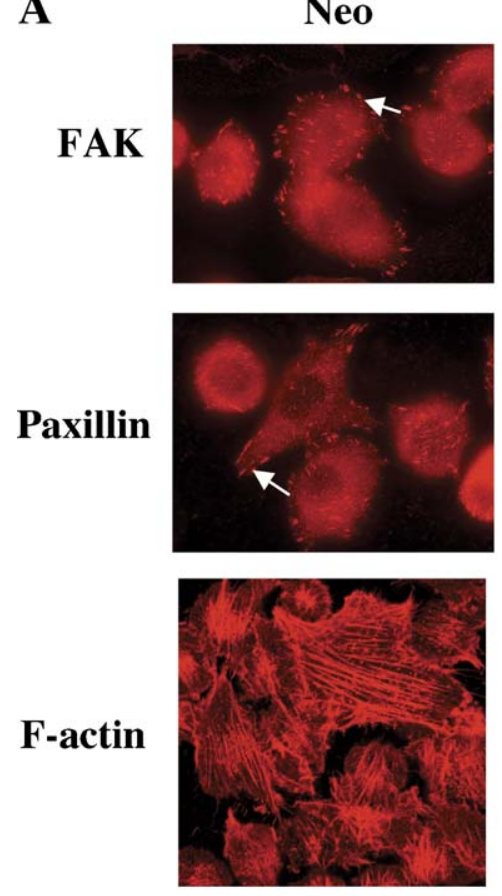

FL
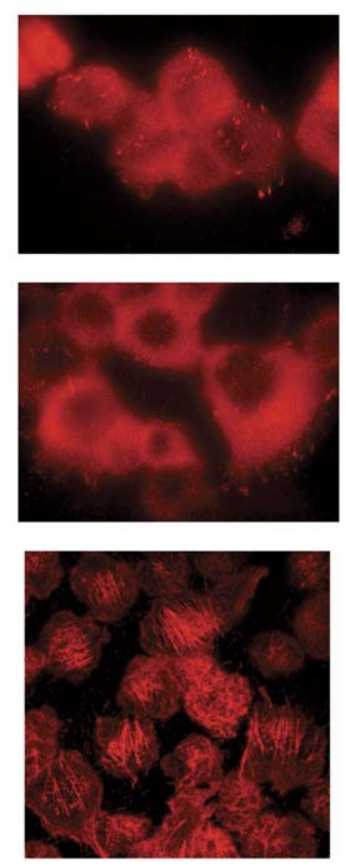
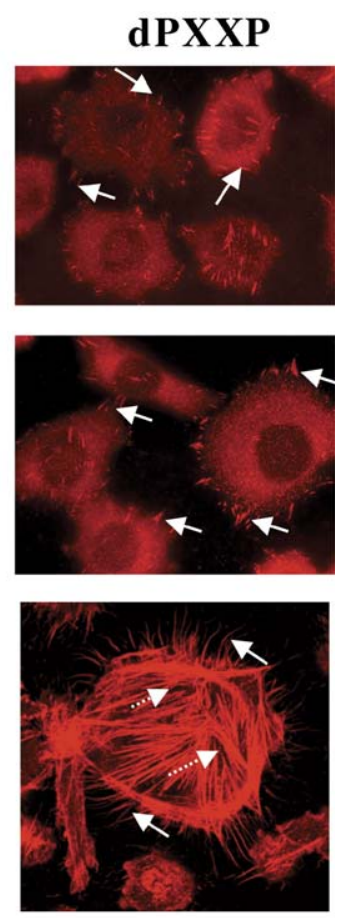

B

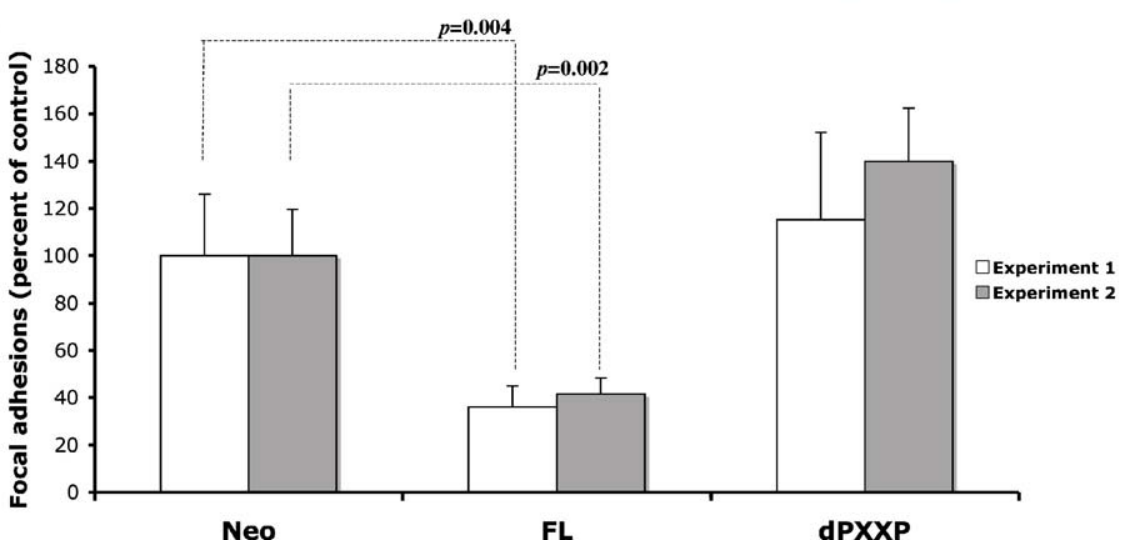

Fig. 4 - Reduced frequency of focal adhesions, stress fibers, and filopodia in FL cells. (A) Immunofluorescence: Top and center rows: cells were plated on collagen IV-coated coverslips and allowed to attach for $3 \mathrm{~h}$. Cells were then fixed, permeabilized, and probed with either anti FAK or anti paxillin antibody. Shown is a representative of at least 3 experiments; arrows point to focal adhesions. Magnification =100x. Bottom row: cells were grown on collagen IV for $3 \mathrm{~h}$, fixed, permeabilized, and probed with rhodamine-phalloidin antibody. Solid arrows indicate filopodia, dashed arrows point to stress fibers. Shown are representatives of at least 3 experiments. Magnification $=100 \times$. (B) Focal adhesion quantification: 5 random fields per coverslip of FAK antibody-stained Neo, FL, or dPXXP cells were photographed under a confocal microscope. Focal adhesions were counted randomly on up to 12 cells and averaged to indicate number of focal adhesions per cell. Shown are the results of two separate experiments, \pm SD. 
collagen IV-coated filters. FL cell migration to serum was significantly reduced compared to Neo ( $14 \%$ of control, $p=0.0004$; Fig. 2). However, migration of dPXXP cells under identical conditions was augmented (228\%, $p=0.00001)$. Inhibition of migration of FL cells compared to Neo was less affected by gene expression in the absence of attractant (73\%, $p=0.006$; Fig. 2), whereas there was no significant difference between basal levels of migration of dPXXP and Neo cells under BSA-only conditions. Migration of Neo cells to serum was $30 \%$ reduced compared to their baseline migration to $0.1 \%$ BSA $(p=0.01)$, whereas migration of $\mathrm{FL}$ cells to serum was reduced by $87 \%$ compared to their migration to $0.1 \%$ BSA $(p=0.0001)$. Migration of dPXXP cells to serum was increased by $174 \%$ compared to dPXXP cell migration to $0.1 \%$ BSA ( $p=0.00001)$.

Cell migration involves formation of focal adhesions to anchor the leading edge of the cell [1]. We tested whether adhesion of the cell sublines to collagen IV mirrored the results of the migration assays. Significantly fewer FL cells attached to collagen type IV in a serum background ( $p=0.03$; Table 1 , Fig. $3 A)$. Adhesion of FL cells to collagen I, fibronectin, vitronectin, and laminin was also reduced (Table 1). dPXXP cells attached in significantly greater numbers than Neo cells to substrate in the presence of serum $(p=0.01)$. This suggests that overexpression of CAIR-1 reduces migration of cells to serum, at least in part, through decreased adhesion to substrate, and that the role of CAIR-1 in adhesion is not necessarily substratum specific. Both attachment and migration require dynamic focal adhesion disassembly and rear-end detachment $[1,19]$. Studies have illustrated the role of the calciumdependent protease, calpain, on focal adhesion disassembly and migration $[18,20]$. Calpain activity was assessed to ask whether increased detachment plays a role in the decreased adhesion of FL cells. Although virtually $100 \%$ of cells fluoresced indicating active calpain, there was no discernible difference in relative fluorescence between FL, dPXXP, and Neo cells (Fig. 3B). This argues that the rates of cell detachment may not differ markedly with changes in CAIR-1. These data suggest that the differences in migration and adhesion are more likely caused by a lack of initial attachment to substratum.

Focal adhesions are reduced in FL cells

Focal adhesion signalosomes are required for motility, attachment, and spreading of cells [21]. The subcellular localization of focal adhesion proteins is important for efficiency and rate of attachment and migration [22]. Cells attached to collagen IV were stained for FAK and paxillin to assess relative quantity and cellular distribution of focal adhesions. Fig. 4A shows a redistribution of FAK to the periphery of APXXP cells compared to Neo control or FL cells. Quantification of these focal adhesions showed a significant decrease in FL cells compared to Neo and dPXXP (decreased by 64\%, $p=0.004$ and 59\%, $p=0.002$ compared to Neo; $69 \%, p=0.007$ and $70 \%, p=0.0003$ compared to dPXXP; Fig. 4B). Paxillin is a key partner protein of FAK $[12,23]$, being a substrate of FAK that enhances downstream signaling in migration pathways. The pattern of focal adhesions observed by paxillin staining was similar to that of FAK, more peripheral in dPXXP cells whereas markedly

decreased in number in FL cells (Fig. 4A). The difference in focal adhesion organization and abundance suggested that the cytoskeletal organization was different, especially as the actin

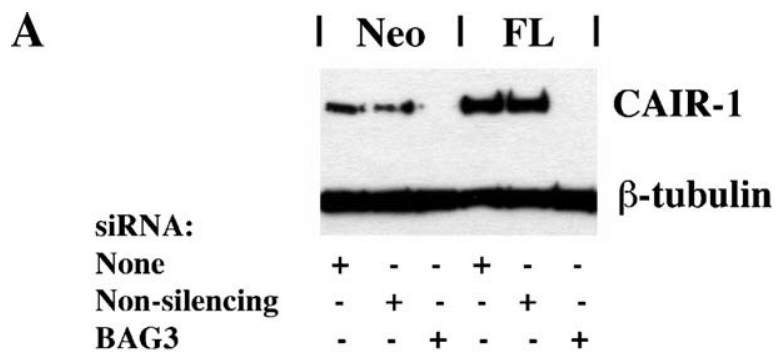

B

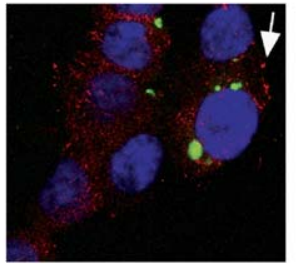

Red:

Green: Non-silencing siRNA

Blue:

C

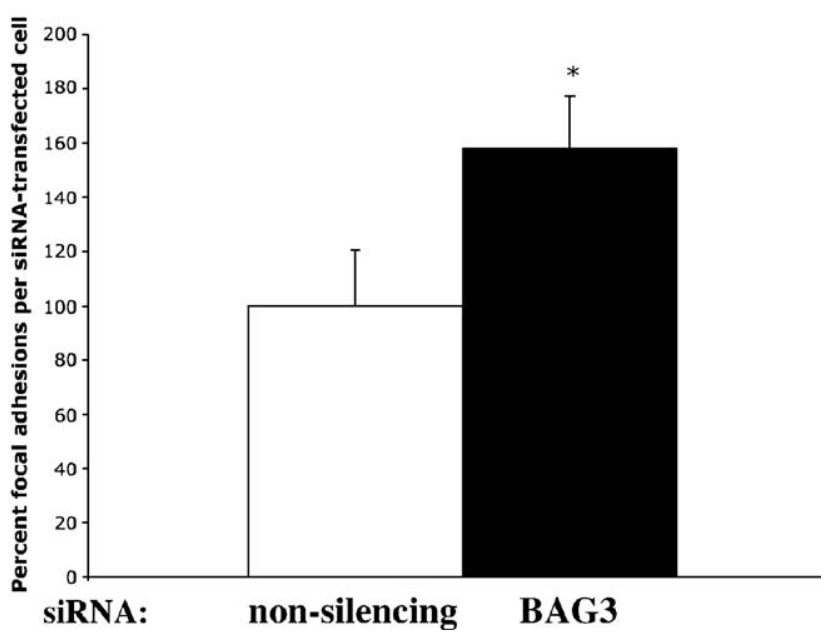

Fig. 5 - Gene silencing of CAIR-1 increases focal adhesions in FL cells. (A) Western blot of Neo and FL cells treated with non-silencing or BAG-3 siRNA labeled with Alexa Fluor 488 (40nM) or no siRNA. Cells were treated with siRNA for $48 \mathrm{~h}$, following which the cell lysates were separated by SDS-PAGE and immunoblotted with anti CAIR-1 antibody. $\beta$-Tubulin was used as a loading control. (B) FL cells treated with non-silencing or BAG-3 siRNA labeled with Alexa Fluor 488 (green) for $48 \mathrm{~h}$ and allowed to attach to collagen IV-coated coverslips for $3 \mathrm{~h}$. Focal adhesions were stained with FAK antibody and Alexa Fluor 594-conjugated rabbit anti-mouse secondary antibody (red). Arrows indicate focal adhesions. Magnification $=100 \times$. (C) Average focal adhesions per cell as determined by quantitation of focal adhesions in FAK-stained FL cells treated with siRNA; only green cells were evaluated in five fields per coverslip. ${ }^{*} p=0.0005, \pm$ SEM, $n=3$. 
cytoskeleton is critical in modulating cell adhesion and migration [24]. Cells were stained with rhodamine-phalloidin to elucidate cytoskeletal organization. F-actin stress fibers were common in dPXXP cells (Fig. 4) but were reduced in FL cells. Furthermore, dPXXP cells exhibited plentiful and thicker filopodial protrusions compared to Neo (arrows; Fig. 4A). These results show that deletion of the CAIR-1 SH3binding domain in cells is associated with formation of more peripheral and stronger focal adhesions and a different cytoskeletal organization than cells overexpressing FL CAIR-1.

CAIR-1 gene silencing in FL cells increases focal adhesions

As overexpressing CAIR-1 leads to decreased focal adhesions, we tested whether CAIR-1 was directly responsible for this phenotype by silencing the CAIR-1 gene via RNAi. We treated FL cells with Alexa Fluor 488 (green)-labeled BAG-3 siRNA or control (non-silencing) siRNA for $48 \mathrm{~h}$, which was determined to be sufficient to reduce protein expression (Fig. 5A). Cells were then allowed to attach to collagen IV-coated coverslips for $3 \mathrm{~h}$, after which they were fixed and stained with anti-FAK antibody. Focal adhesions on FL cells transfected with the BAG-3 siRNA were generally clearer and more abundant (Fig. 5B). We quantitated the number of focal adhesions and found that the average focal adhesions per cell in BAG-3 siRNA- transfected cells was $158 \%(p=0.0005)$ of that in cells transfected with non-silencing siRNA (Fig. 5C).

CAIR-1 does not localize with focal adhesions in FL cells

The reduction in focal adhesions in FL cells led to the question of the dynamics of CAIR-1 localization. Dual staining for focal adhesions and CAIR-1 was evaluated using confocal imaging (Fig. 6). We found abundant FAK-CAIR-1 co-localization predominantly in the cytoplasm, especially in FL cells. CAIR-1 was largely absent from focal adhesion points at the periphery of the cells. We tested for direct interaction between CAIR-1 and focal adhesion proteins (including paxillin, src, and others) by immunoprecipitation or GSTPXXP domain pull-down experiments and found no such direct interaction (data not shown). These results suggest that CAIR-1 modulates focal adhesion signaling remotely from the cytoplasm.

\section{Phosphorylation of FAK and paxillin is reduced in FL cells}

Focal adhesion signalosomes are comprised of many activated proteins. Most signalosome components have protein-protein interactions, including SH3 interactions, for downstream signal propagation $[25,26]$. The marked difference observed in focal adhesion quantity and location suggested altered modulation at the level of signal transduction. Phosphory-
Neo

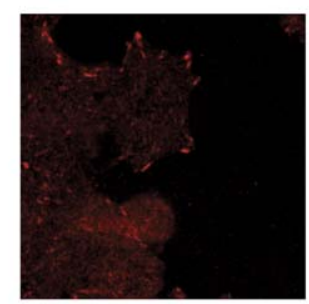

FAK

CAIR-1

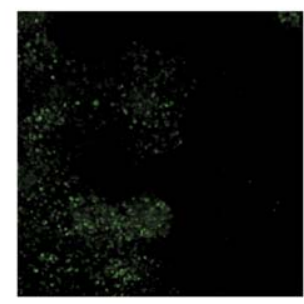

\section{Combined}

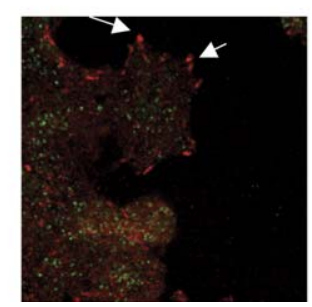

FL
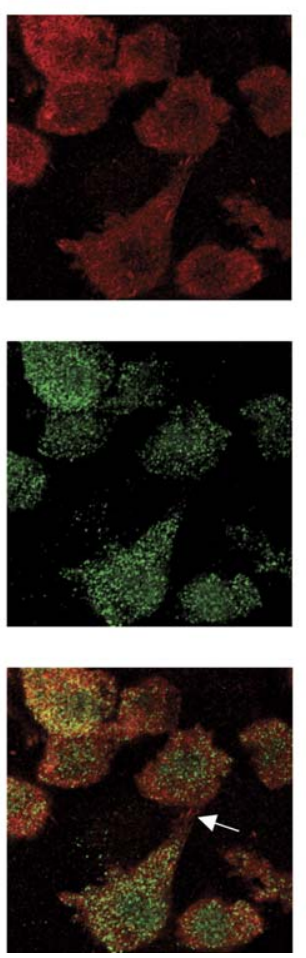

dPXXP
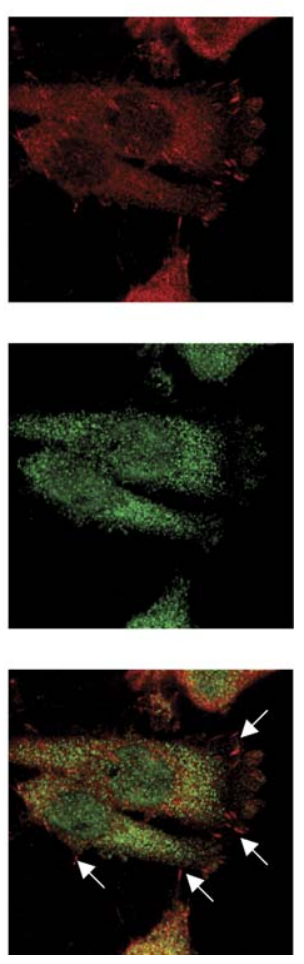

Fig. 6 - CAIR-1 does not co-localize with FAK to focal adhesion points. (A) Cells were grown on collagen type IV for $3 \mathrm{~h}$ after which they were fixed and probed with FAK and CAIR-1 antibodies. FAK was visualized with Alexa-Fluor 594 secondary antibody (red), whereas CAIR-1 was visualized with Alexa-Fluor 488 secondary antibody (green). Arrows point to focal adhesions. Yellow indicates areas of co-localization. Magnification $=100 x$. 
A
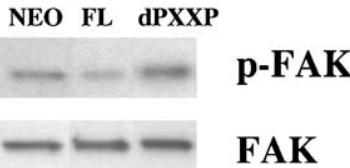

B

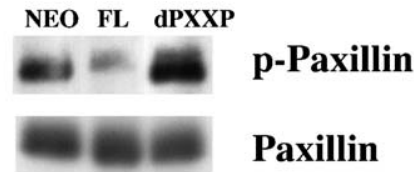

C
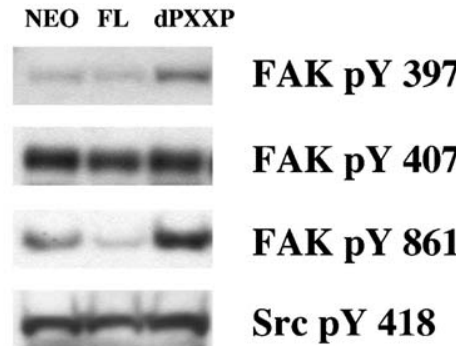

Fig. 7 - Overexpression of FL CAIR-1 reduces phosphorylation of FAK and paxillin. Lysates of cells grown on collagen IV overnight were subjected to immunoprecipitation with anti-phosphotyrosine antibody and probed with either anti-FAK (A) or anti-paxillin (B). Total FAK and paxillin protein blots are shown immediately below the phosphoprotein levels, respectively. (C) Antibodies specific to phosphorylated $\mathrm{Y}^{397}, \mathrm{Y}^{407}$, and $\mathrm{Y}^{861}$ on $\mathrm{FAK}$ and $\mathrm{Y}^{418}$ on Src were used to detect phosphorylation in lysates of Neo, FL, or dPXXP cells grown overnight on collagen IV. Forty micrograms of protein was used for Western analyses.

lation of FAK was diminished in FL cells (Fig. 7A), whereas in dPXXP cells p-FAK was increased above that of Neo control. Total FAK protein quantities were unchanged across the sublines. Paxillin phosphorylation patterns mirrored those of FAK (Fig. 7B), where p-paxillin was weaker in FL cells and stronger in dPXXP cells. Total paxillin quantities also were unchanged. We tested the possibility that CAIR-1 may activate a tyrosine phosphatase by comparing migration and p-FAK levels in cells treated with the phosphotyrosine phosphatase inhibitor sodium vanadate. Vanadate treatment did not alter motility or relative phosphorylation events (data not shown), making it unlikely that CAIR-1 modulation of adhesion proteins is via phosphotyrosine phosphatase activation.

FAK has multiple tyrosine residues, for which selective antibodies are now available. Phosphorylation of the primary catalytic site $\mathrm{Y}^{397}$, as well as of $\mathrm{Y}^{861}$, both of which are strong Srcmodulated sites $[27,28]$, was markedly reduced in FL cells butnot in dPXXP cells. However, phosphorylation of $\mathrm{Y}^{407}$, associated with VEGFR signaling but is not a Src substrate [29], was relatively unchanged (Fig. 7C). These results suggest that modulation of adhesion proteins by CAIR-1 may be Src specific. We tested Src activity among cell lines by probing for phosphoSrc at the activation-specific $Y^{418}$ residue (Fig. 7C). Unlike FAK and paxillin, there was no visible difference in p-Src levels among the cell lines, suggesting that CAIR-1-negative modulation may occur by interfering with Src downstream interactions. However, Src does not co-immunoprecipitate with CAIR-1 nor does a GST-Src-SH3 fusion protein domain pull-down CAIR-1 (data not shown), indicating that such modulation is likely indirect.

CAIR-1 overexpression reduces FAK interaction with downstream signaling proteins $p^{130} \mathrm{Cas}$ and Crk

We tested FAK binding to its downstream partner protein $\mathrm{p}^{130}$ Cas in order to assess the downstream effect of CAIR-1 on FAK/paxillin/src activity. $\mathrm{p}^{130} \mathrm{Cas}$ is an SH3-containing protein that is involved in focal adhesion and actin assembly [30]. FAK co-immunoprecipitation with $\mathrm{p}^{130} \mathrm{Cas}$ was reduced in FL cells but not in dPXXP cells, following the pattern seen for FAK and paxillin phosphorylation (Fig. 8A). Furthermore, $\mathrm{p}^{130}$ Cas association with its downstream partner Crk was also reduced in FL cells, but not in dPXXP cells (Fig. 8B), reasserting the negative modulation characteristics of CAIR-1, and attributing this to its ability to interact through its proline-rich domain. However, CAIR-1 does not directly bind to $\mathrm{p}^{130}$ Cas or Crk (data not shown).

\section{Discussion}

We hypothesized that CAIR-1 regulates cell adhesion and migration signaling pathways through interaction with SH3 domain-containing proteins via proline-rich (PXXP) domain interactions. We found that overexpressing FL CAIR-1 in MDA435 breast carcinoma cell lines results in reduced migration, adhesion, and focal adhesion formation and signaling. However, overexpressing a CAIR-1 mutant incapable of SH3 protein binding paradoxically strengthened migration and adhesion. Theloss of migration to serum through a collagen type IV-coated filter was of interestbecause this differential migration to serum was not observed when using EGF or IGF-1 as chemoattractants, suggesting a signaling mechanism independent of these
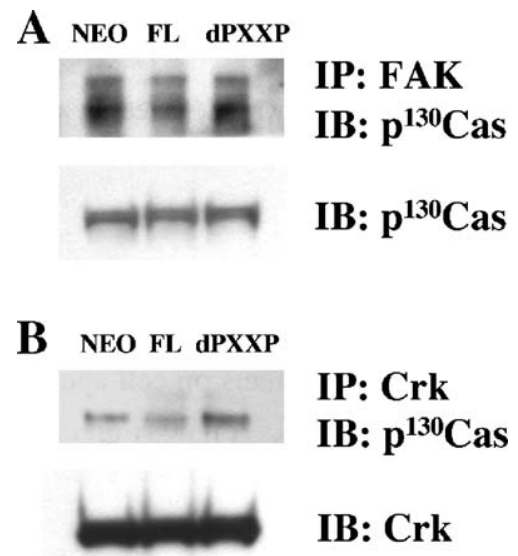

Fig. 8 - Overexpression of FL CAIR-1 decreases FAK association with downstream proteins $\mathrm{p}^{130}$ Cas and Crk. (A) Lysates of Neo, FL, and dPXXP cells grown overnight on collagen IV were immunoprecipitated with anti-FAK antibody and transferred membranes were immunoblotted against anti- $\mathrm{p}^{130}$ Cas antibody. (B) Lysates were immunoprecipitated with anti-Crk antibody and membranes were immunoblotted against anti $\mathrm{p}^{130}$ Cas antibody. 
ligands. Cells overexpressing CAIR-1 also exhibit reduced phosphorylation of two key focal adhesion proteins, FAK and paxillin, whereas deletion of the CAIR-1 PXXP domain resulted in an overactivation of phospho-FAK and phospho-paxillin levels that exceeded those of Neo control. The tyrosine residues on FAK that were differentially phosphorylated implicate Src in the activated pathway. The effects of CAIR-1 on spreading and migration were observed at upstream points in the signal transduction hierarchy. These results point to a novel regulatory mechanism for the stress protein CAIR- 1 in focal adhesion development and maintenance.

Our data suggest a unique regulatory role for protein interactions with the PXXP domain of CAIR-1. The importance of protein-SH3 domain interactions in adhesion and migration pathways is well documented, and they are usually associated with activation or inhibition of kinases and recruitment of downstream signaling components [14,25,31]. The phenotypes associated with CAIR-1 overexpression or PXXP deletion may be the result of CAIR-1 inhibiting focal adhesion proteins either by sequestration of such proteins in the cytosol or by impairment of the downstream proteins' ability to associate with their partners. Our observation that both FAK and CAIR-1 are primarily cytosolic in FL cells hints in this direction. Another mode of action may involve CAIR-1activating inhibitory proteins that cause downregulation of attachment and migration. Dysregulation of a tyrosine phosphatase through one of these mechanisms seems unlikely, as treating cells with sodium vanadate did not stabilize FAK phosphorylation in dPXXP cells or cause these cells to adopt FL cell-like phenotypes. Although previous studies showed that CAIR-1 binds inactive PLC $\gamma$, a protein implicated in cell migration, and is then released upon EGF stimulation [6,32-34], we observed no differential effect of EGF treatment on FAK and paxillin phosphorylation or cell motility in this system. We further treated our cell sublines with the PLC inhibitor U73122 to test for a potential role for PLC $\gamma$ in our observed phenotypes. No significant effect of this drug on FAK phosphorylation or cell adhesion to collagen IV was demonstrated (data not shown).

CAIR-1 overexpression resulted in decreased phosphorylation of $\mathrm{Y}^{397}$ and $\mathrm{Y}^{861}$ FAK, both of which are major Srcassociated phosphorylation sites $[27,28]$. Binding of FAK with its downstream partner proteins $\mathrm{p}^{130} \mathrm{Cas}$ and Crk was reduced in FL cells. These results are opposite those in dPXXP cells. Association of $\mathrm{p}^{130} \mathrm{Cas}$ and Crk with FAK involves upstream signaling between FAK, paxillin, and Src $[15,26,35]$. Thus, these data point to a possible negative modulation of FAK/Src interaction by the CAIR-1 proline-rich domain. This effect then inhibits select downstream interactions of FAK because phosphorylation of Src on its activation site $\mathrm{Y}^{418}$ was not altered in FL or dPXXP cells. However, immunofluorescence studies showed that CAIR-1 did not localize to the focal adhesion, and CAIR-1 also did not co-immunoprecipitate with Src or paxillin. These data together suggest that although CAIR-1 modulation may occur distally through a mechanism not yet established, it produces pathway-wide effects on cell signaling of adhesion and migration.

Our data showing significantly reduced migration, adhesion, and formation of focal adhesions in FL cells argues that adhesion may be the initial required event for CAIR-1 modulated cell-ECM interaction in response to serum, through which the migratory phenotypes are then expressed. One explanation is that certain serum growth factors may differentially induce cell adhesion as a function of CAIR-1 expression. Studies have also shown that growth factors such as EGF can substitute for serum in stimulation of directional migration in selected cells [36]. However, neither EGF nor IGF-1 alone mirrored the effect of serum in inducing dPXXP or inhibiting FL migration. These data also suggest that integrins may indirectly have a role in the differential behavior of FAK and paxillin in the MDA-435 sublines. Integrins are stimulated by their interaction with the ECM and relay signals downstream to FAK, paxillin, and other focal adhesion proteins [24,37-39]. No differences in total proteins levels of integrin $\beta 1$ or integrinlinked kinase (ILK) were found in our cell sublines. Furthermore, the observation that FAK activity is reduced in FL cells and that this reverberates down the $\mathrm{p}^{130} \mathrm{Cas} / \mathrm{Crk}$ pathway strongly suggests that CAIR-1 modulation of adhesion and migration is occurring upstream. It remains to be determined whether a missing SH3 protein-containing link is responsible for the observed phenotypes or whether cytoplasmic CAIR-1 has an overall impact on the cell signaling machinery.

In summary, we have demonstrated that a distinct binding region on CAIR-1, the proline-rich region, negatively regulates activation of cell adhesion and migration pathway proteins in an indirect fashion. This provides a new perspective of the processes that govern tumor cell migration, including assembly and dissolution of the focal adhesion points, by demonstrating the modulation of these processes by the calcium influx-regulated stress protein CAIR-1/BAG-3. Such findings provide a further avenue of approaching dysregulated signaling in cancer by designing interventions against indirect signaling partners, such as CAIR-1, that can alter cell phenotype.

\section{Acknowledgments}

The authors thank A. Murphy, L. Harford, and J. Saltsman for their technical assistance.

This research was supported by the Intramural Research Program of the NIH, National Cancer Institute, Center for Cancer Research.

\section{REFEREN C E S}

[1] D.A. Lauffenburger, A.F. Horwitz, Cell migration: a physically integrated molecular process, Cell 84 (1996) 359-369.

[2] L.A. Liotta, E.C. Kohn, The microenvironment of the tumour-host interface, Nature 411 (2001) 375-379.

[3] A. Wells, Tumor invasion: role of growth factor-induced cell motility, Adv. Cancer Res. 78 (2000) 31-101.

[4] J. Kassis, D.A. Lauffenburger, T. Turner, A. Wells, Tumor invasion as dysregulated cell motility, Semin. Cancer Biol. 11 (2001) 105-117.

[5] H. Doong, K. Rizzo, S. Fang, V. Kulpa, A.M. Weissman, E.C. Kohn, CAIR-1/BAG-3 abrogates heat shock protein-70 chaperone complex-mediated protein degradation: accumulation of poly-ubiquitinated Hsp90 client proteins, J. Biol. Chem. 278 (2003) 28490-28500. 
[6] H. Doong, J. Price, Y.S. Kim, C. Gasbarre, J. Probst, L.A. Liotta, J. Blanchette, K. Rizzo, E. Kohn, CAIR-1/BAG-3 forms an EGF-regulated ternary complex with phospholipase C-gamma and Hsp70/Hsc70, Oncogene 19 (2000) 4385-4395.

[7] C.C. Felder, A.L. Ma, L.A. Liotta, E.C. Kohn, The antiproliferative and antimetastatic compound L651582 inhibits muscarinic acetylcholine receptor-stimulated calcium influx and arachidonic acid release, J. Pharmacol. Exp. Ther. 257 (1991) 967-971.

[8] E.C. Kohn, C.C. Felder, W. Jacobs, K.A. Holmes, A. Day, R. Freer, L.A. Liotta, Structure-function analysis of signal and growth inhibition by carboxyamido-triazole, CAI, Cancer Res. 54 (1994) 935-942.

[9] H. Doong, A. Vrailas, E.C. Kohn, What's in the 'BAG'?-A functional domain analysis of the BAG-family proteins, Cancer Lett. 188 (2002) 25-32.

[10] J. Brabek, S.S. Constancio, P.F. Siesser, N.Y. Shin, A. Pozzi, S.K. Hanks, Crk-associated substrate tyrosine phosphorylation sites are critical for invasion and metastasis of SRC-transformed cells, Mol. Cancer Res. 3 (2005) 307-315.

[11] M. Vidal, V. Gigoux, C. Garbay, SH2 and SH3 domains as targets for anti-proliferative agents, Crit. Rev. Oncol./Hematol. 40 (2001) 175-186.

[12] D.D. Schlaepfer, C.R. Hauck, D.J. Sieg, Signaling through focal adhesion kinase, Prog. Biophys. Mol. Biol. 71 (1999) 435-478.

[13] M.D. Schaller, Biochemical signals and biological responses elicited by the focal adhesion kinase, Biochim. Biophys. Acta 1540 (2001) 1-21.

[14] J.T. Parsons, Focal adhesion kinase: the first ten years, J. Cell Sci. 116 (2003) 1409-1416.

[15] M.P. Playford, M.D. Schaller, The interplay between Src and integrins in normal and tumor biology, Oncogene 23 (2004) 7928-7946.

[16] S.K. Hanks, L. Ryzhova, N.Y. Shin, J. Brabek, Focal adhesion kinase signaling activities and their implications in the control of cell survival and motility, Front. Biosci. 8 (2003) d982-d996.

[17] E.W. Thompson, S. Paik, N. Brunner, C.L. Sommers, G. Zugmaier, R. Clarke, T.B. Shima, J. Torri, S. Donahue, M.E. Lippman, G.R. Martin, R.B. Dickson, Association of increased basement membrane invasiveness with absence of estrogen receptor and expression of vimentin in human breast cancer cell lines, J. Cell. Physiol. 150 (1992) 534-544.

[18] A. Glading, P. Chang, D.A. Lauffenburger, A. Wells, Epidermal growth factor receptor activation of calpain is required for fibroblast motility and occurs via an ERK/MAP kinase signaling pathway, J. Biol. Chem. 275 (2000) 2390-2398.

[19] P. Friedl, E.B. Brocker, The biology of cell locomotion within three-dimensional extracellular matrix, Cell. Mol. Life Sci. 57 (2000) 41-64.

[20] N. Dourdin, A.K. Bhatt, P. Dutt, P.A. Greer, J.S. Arthur, J.S. Elce, A. Huttenlocher, Reduced cell migration and disruption of the actin cytoskeleton in calpain-deficient embryonic fibroblasts, J. Biol. Chem. 276 (2001) 48382-48388.

[21] B. Wehrle-Haller, B. Imhof, The inner lives of focal adhesions, Trends Cell Biol. 12 (2002) 382-389.

[22] G. Maheshwari, G. Brown, D.A. Lauffenburger, A. Wells, L.G. Griffith, Cell adhesion and motility depend on nanoscale RGD clustering, J. Cell Sci. 113 (Pt. 10) (2000) 1677-1686.

[23] M.D. Schaller, Paxillin: a focal adhesion-associated adaptor protein, Oncogene 20 (2001) 6459-6472.
[24] A.J. Ridley, M.A. Schwartz, K. Burridge, R.A. Firtel, M.H. Ginsberg, G. Borisy, J.T. Parsons, A.R. Horwitz, Cell migration: integrating signals from front to back, Science 302 (2003) 1704-1709.

[25] D.J. Sieg, C.R. Hauck, D.D. Schlaepfer, Required role of focal adhesion kinase (FAK) for integrin-stimulated cell migration, J. Cell Sci. 112 (Pt. 16) (1999) 2677-2691.

[26] T.R. Polte, S.K. Hanks, Interaction between focal adhesion kinase and Crk-associated tyrosine kinase substrate p130Cas, Proc. Natl. Acad. Sci. 92 (1995) 10678-10682.

[27] M.D. Schaller, J.D. Hildebrand, J.D. Shannon, J.W. Fox, R.R. Vines, J.T. Parsons, Autophosphorylation of the focal adhesion kinase, pp125FAK, directs SH2-dependent binding of pp60src, Mol. Cell. Biol. 14 (1994) 1680-1688.

[28] M.B. Calalb, X. Zhang, T.R. Polte, S.K. Hanks, Focal adhesion kinase tyrosine-861 is a major site of phosphorylation by Src, Biochem. Biophys. Res. Commun. 228 (1996) 662-668.

[29] F. LeBoeuf, F. Houle, J. Huot, Regulation of vascular endothelial growth factor receptor 2-mediated phosphorylation of focal adhesion kinase by heat shock protein 90 and Src kinase activities, J. Biol. Chem. 279 (2004) 39175-39185.

[30] Y. Nojima, N. Morino, T. Mimura, K. Hamasaki, H. Furuya, R. Sakai, T. Sato, K. Tachibana, C. Morimoto, Y. Yazaki, H. Hirai, Integrin-mediated cell adhesion promotes tyrosine phosphorylation of p130Cas, a Src homology 3-containing molecule having multiple Src homology 2-binding motifs, J. Biol. Chem. 270 (1995) 15398-15402.

[31] J.W. Thomas, B. Ellis, R.J. Boerner, W.B. Knight, G.C. White II, M.D. Schaller, SH2- and SH3-mediated interactions between focal adhesion kinase and Src, J. Biol. Chem. 273 (1998) 577-583.

[32] J. Kassis, J. Moellinger, H. Lo, N.M. Greenberg, H.G. Kim, A. Wells, A role for phospholipase C-gamma-mediated signaling in tumor cell invasion, Clin. Cancer Res. 5 (1999) 2251-2260.

[33] P. Chen, H. Xie, M.C. Sekar, K. Gupta, A. Wells, Epidermal growth factor receptor-mediated cell motility: phospholipase $\mathrm{C}$ activity is required, but mitogen-activated protein kinase activity is not sufficient for induced cell movement, J. Cell Biol. 127 (1994) 847-857.

[34] A. Kamat, G. Carpenter, Phospholipase C-gamma1: regulation of enzyme function and role in growth factor-dependent signal transduction, Cytokine Growth Factor Rev. 8 (1997) 109-117.

[35] D.J. Webb, K. Donais, L.A. Whitmore, S.M. Thomas, C.E. Turner, J.T. Parsons, A.F. Horwitz, FAK-Src signalling through paxillin, ERK and MLCK regulates adhesion disassembly, Nat. Cell Biol. 6 (2004) 154-161.

[36] E.G. Bade, B. Nitzgen, Extracellular matrix (ECM) modulates the EGF-induced migration of liver epithelial cells in serum-free, hormone-supplemented medium, In Vitro Cell. Dev. Biol. 21 (1985) 245-248.

[37] J.D. Hood, D.A. Cheresh, Role of integrins in cell invasion and migration, Nat. Rev., Cancer 2 (2002) 91-100.

[38] C.E. Turner, Paxillin and focal adhesion signalling, Nat. Cell Biol. 2 (2000) E231-E236.

[39] D.D. Schlaepfer, S.K. Mitra, Multiple connections link FAK to cell motility and invasion, Curr. Opin. Genet. Dev. 14 (2004) 92-101. 